\title{
An Empirical Research on the Structure of Public Expenditure and Economic Growth-Evidence from China
}

\author{
Li Yang $\mathrm{Zi}^{1}$ \\ ${ }^{1}$ Department of Urban Planning, Tsinghua University, Beijing, China \\ Correspondence: Li Yang Zi, Department of Urban Planning, Tsinghua University, Beijing, 100084, China. \\ E-mail: liyangzi@mail.tsinghua.edu.cn
}

Received: January 5, 2016

Accepted: January 29, 2016

Online Published: February 25, 2016

doi:10.5539/ijef.v8n3p85

URL: http://dx.doi.org/10.5539/ijef.v8n3p85

\begin{abstract}
This paper takes the relationship between public expenditure structure and economic growth as the object of study. By using evidence from China, it analyzes how the Chinese Government should promote economic growth by the optimization of public expenditure structure when facing with a decline in the growth of fiscal revenue. The main conclusions of the paper are as follows: The impact of public expenditure structure on economic growth not only depends on the output elasticity of the item of expenditure, but also on its initial share. When the initial share is oversized, it will make an item of expenditure which appears to be productive become non-productive. For China, the proportions of economic construction expenditure and administrative expenditure have a significant negative effect on long-term economic growth; the social expenditure on culture and education has a significant positive effect on long-term economic growth.
\end{abstract}

Keywords: structure of public expenditure, economic growth, economic construction expenditure, social expenditure on culture and education, defense expenditure, administrative expenditure

\section{Introduction}

Since the economic crisis in 2008, the economic growth of global major economies has generally slowed down. The world's economy has entered into a phase of deep adjustment. Along with the economic downturn, the growth rate of fiscal revenue has inevitably slowed down. Taking China as an example, in 2014 the general public revenue nationwide increased by 8.6\%, a drop of 1.6 and 4.3 percentage points compared with 2013 and 2012 respectively. And it was a fall of 16.4 percentage points when compared with $25 \%$ of 2011 . Under the constraint of tight fiscal revenue, the pattern which drives economic growth by large-scale fiscal expansion cannot be maintained. Therefore with a certain amount of total public expenditure, the key to implementing current fiscal policies is to promote the increase of economic growth efficiency and thereby push forward the economic growth by adjusting the ratio of the public expenditure structure. And to optimize the public expenditure structure, in-depth study must be made on the relationship between public expenditure structure and economic growth, so as to estimate, in the current economic situation, which items of expenditure should be cut and which should be added.

This paper takes the relationship between public expenditure structure and economic growth as the object of study. Section II is the related literatures review. In Section III a theoretical model of the impact of public expenditure structure on economic growth is established. In Section IV, empirical test is provided. Based on evidence from China, we make analysis of the dynamic relationship between public expenditure structure and economic growth and its time trajectory and test which items of expenditure have a positive effect and which have a negative effect on long-term economic growth. Explanations of empirical test results are provided in Section V. Section VI presents conclusions and policy suggestions.

\section{Related Literatures}

In 1970s, Arrow and Kruz (1970) introduced public expenditure into mathematical model, emphasizing that the representative individual can gain utility from public expenditure and representative firms can also benefit from public expenditure. Since then, many scholars (Chen, 2006; MacDonald, 2008; Woodford, 2011) have studied the relationship between public expenditure and economic growth. Barro(1990), as the typical representative, has built and improved the theoretical model of the relationship between productive expenditure and the economy, 
based on endogenous growth model. He pointed out that the relationship between the proportion of productive expenditure and economic growth rate is an inverted $U$ type, i.e., the productive expenditure has an optimal size, when the share of productive expenditure is beyond the optimal size, it will have a negative effect on economic growth, but if the share has not yet reached the optimal size, the society can promote economic growth by the increase of productive expenditure.

In the empirical research, Barro (1991) made analysis of economic growth theory by utilizing evidence of 1960 to 1985 from 98 countries. He divided government expenditure into two types: one is government consumption and the other is government investment. The research came up with the following conclusions: Economic growth has a negative correlation with the proportion of government consumption in GDP and its relationship with government investment is non-significant. Shantayanan Devarajan,Vinaya Swaroop and Heng-fu Zou (1996) analyzed the relationship between public expenditure structure and economic growth, using evidence of 20 years from 43 developing countries. They concluded that for developing countries, the proportion of capital expenditure in Total expenditure has a negative effect on economic growth and the proportion of current expenditure in Total expenditure has a positive effect on economic growth. Since then, more and more researches (Blankenau, 2007; Velázquez, 2004; Sanz, 2011) have focused on the evidence from OECD counties and developing countries. For example, using evidence from 9 developing countries in Latin America, Ramirez and Nazmi (1997) concluded that consumption expenditure has a negative effect on economic growth and educational, medical and health care expenditure have a positive effect on economic growth. Zheng (2012), using the panel data of 1997 to 2009 of china's west area, east area and middle area, examined the economic growth effect of public expenditure structure between different areas, and others (Gao, 2012), using time series data from 1996 to 2006, examined the economic growth effect of public expenditure structure.

From the above literature review, we can see that there are many researches on the effect of public expenditure structure on economic growth. However, regarding which items of expenditure are productive, i.e. those having a positive effect on long-term economic growth; and which are consumption expenditure, i.e. those having a negative effect on long-term economic growth, the consensus have not been reached yet. Sometimes there are even opposite conclusions. The reasons for this divergence could be the differences in research objects (countries/regions), research data and research methods. Meanwhile, the existing researches have the following characteristics: Firstly, they are mainly based on empirical studies and lack rigorous theoretical model; secondly, they are mainly based on static analysis to study the long-term effect of public expenditure structure on economic growth. In light of that, this paper, first of all, establish a theoretical model to analyze and discuss theoretically the relationship between public expenditure structure and economic growth. Then we adopt dynamic analysis method to improve the issue of inconsistent research results caused by the lack of analysis on the dynamic time path of the relationship between public expenditure structure and economic growth. Thirdly, the paper studies situation in China by using evidence from China.

\section{Theoretical Model}

Based on the analysis framework of Barro (1990), this paper introduces production function which includes the variable of public expenditure structure. It extends Shantayanan Devarajan, Vinaya Swaroop and Heng-fu Zuo's (1996) model from one including two components of productive expenditure and non-productive expenditure in analyzing the relationship of public expenditure structure and economic growth to a model including $\mathrm{n}$ components of public expenditure, so as to analyze the relationship of public expenditure structure and economic growth. By increasing the effect of one item of expenditure on economic growth through reducing another item, the model estimates whether the expenditure is productive or non-productive.

We assume that there exist three market entities, i.e. enterprise, individual and government. Enterprises employ the capital and labor provided by families in a market with perfect competition and carry out production activities. Total output is decided by private capital stock $\mathrm{k}$ and the total input of $\mathrm{n}$ items of government expenditure $g_{i}(i=1 \ldots n)$. Obtain the criterion of estimating whether $g_{i}$ is productive or non-productive by model derivation. Assuming that the production function uses the form of constant elasticity of substitution (CES) of the element, the production function is as equation (1) after introducing the variable of public expenditure.

$$
y=f\left(k, g_{1}, g_{2}, \cdots g_{n}\right)=\left[\alpha k^{-\zeta}+\beta_{1} g_{1}^{-\zeta}+\cdots+\beta_{n} g_{n}^{-\zeta}\right]^{-1 / \zeta}
$$

Where $\alpha$ and $\beta_{\mathrm{i}}$ are the partition coefficients, $0<\alpha<1,0<\beta_{\mathrm{i}}<1$ and meets $\alpha+\sum_{\mathrm{i}=1}^{\mathrm{n}} \beta_{\mathrm{i}}=1,-1 \leq \zeta, \quad \zeta$ is substitution parameter.

Government implements balanced budget and adjusts its expenditure by revenue, i.e., government expenditure is 
equal to tax revenue and there is no debt. Government revenue mainly comes from tax revenue collected by a fixed tax rate $\tau$. The total government expenditure $\mathrm{g}$ is distributed to $\mathrm{n}$ items according to different proportions, $\mathrm{g}_{\mathrm{i}}$ means the total amount of expenditure of item $\mathrm{i}$, and $\mathrm{g}_{\mathrm{i}}$ enters into production function to have an impact on economic growth. Equation (2) is the mathematical expression of the balanced budget implemented by government.

$$
g=\tau y
$$

Meanwhile, we introduce the variable of public expenditure structure $\emptyset_{i}$, i.e. the proportion of each public expenditure item $g_{i}$ in total public expenditure, and then we have $0<\emptyset_{i}<1$. The total public expenditure is distributed to $n$ items according to the proportions. Government implements balanced budget and collects tax by a fixed tax rate $\tau$. Combining equation (2), an identical equation depicting public expenditure structure and total public expenditure, tax rate and output can be obtained, as is shown in equation (3):

$$
g=\sum_{i=1}^{n} g_{i} \text { Where } g_{i}=\phi_{i} g=\phi_{i} \tau y
$$

Substituting (3) into (1), we can get the production function including the share of public expenditure. Then we introduce the variable of public expenditure structure into production function, as is shown in equation (4). Moreover, we also assume that government expenditure will not influence the preference of representative consumers, or even the utility they obtain from commodity consumption.

$$
y=\left[a k^{-\zeta}+\sum_{i=1}^{n} \beta_{i} \phi_{i}^{-\zeta} g^{-\zeta}\right]^{-\frac{1}{\zeta}}
$$

Families provide labor and capital to enterprises. Their consumption is decided based on the objective of lifetime utility maximization and thereby, the capital provided to enterprises is also decided. They pay taxes to government according to fixed tax rates and the consumers' expenditure is constrained by their income. Equation (5) shows the present value of the lifetime utility function of representative consumers and the budget constraints on consumption, where $\rho$ is the discount rate of utility and $\tau$ is the fixed tax rate.

$$
\begin{gathered}
U=\int_{0}^{\infty} u(c) e^{-\rho t} d t \\
\text { s.t. } \dot{k}=(1-\tau) y-c
\end{gathered}
$$

And instantaneous utility function as in equation (6) is adopted. We assume that consumers have constant relative risk aversion tendency $\sigma$.

$$
u(c)=\frac{c^{1-\sigma}-1}{1-\sigma}
$$

To solve the pursuit of realizing lifetime utility maximization by representative families is to solve the maximization of social welfare, i.e. to solve equation (7).

$$
\begin{gathered}
\max U=\int_{0}^{\infty} \frac{c^{1-\sigma}-1}{1-\sigma} e^{-\rho t} d t \\
\text { s.t. } \dot{k}=(1-\tau) y-c
\end{gathered}
$$

Firstly, we build the Hamilton function as is shown in equation (8):

$$
\left.H=\frac{c^{1-\sigma}-1}{1-\sigma} e^{-\rho t}+\lambda\left\{(1-\tau)\left(\alpha k^{-\zeta}+g^{-\zeta} \sum_{i=1}^{n} \beta_{i} \phi_{i}^{-\zeta}\right)^{-\frac{1}{\zeta}}\right]-c\right\}
$$

Secondly, maximize Hamilton function by first-order condition, i.e., make the partial derivative of $H(c, \lambda)$ to $c$ as 0 . We can get:

$$
\frac{\dot{c}}{c}=\frac{\frac{1}{\lambda} \frac{d \lambda}{d t}+\rho}{-\sigma}
$$


Thirdly, based on the equation of motion of $\lambda$, solve the differential equation of $\lambda$ and obtain $\lambda$.

$$
\dot{\lambda}=-\frac{\partial H}{\partial k}=-\lambda \alpha(1-\tau)\left[\alpha+\left(\frac{g}{k}\right)^{-\zeta} \sum_{i=1}^{n} \beta_{i} \phi_{i}^{-\zeta}\right]^{-\frac{\zeta+1}{\zeta}}
$$

Then substituting the obtained $\lambda$, or directly, $\lambda$ obtained based on the equation of motion, into equation (9), we can get equation (11).

$$
\frac{\dot{c}}{c}=\frac{\alpha(1-\tau)\left[\alpha+\left(\frac{g}{k}\right)^{-\zeta} \sum_{i=1}^{n} \beta_{i} \phi_{i}^{-\zeta}\right]^{-\frac{(1+\zeta)}{\zeta}}-\rho}{\sigma}
$$

Equation (11) is the Euler equation of representative families' lifetime utility maximization and a deformation of the first-order condition of Hamilton function, describing the growth rate of consumption. It is an equation of motion on consumption, showing the rule that consumption changes along with time.

Besides, we assume $\tau$ as the fixed tax rate, and then get the constant $g / y$ and hence the constant $g / k$, the following can be obtained based on equations (2), (3) and (4):

$$
\frac{g}{k}=\frac{\tau y}{k}=\left[\left(\tau^{\zeta}-\sum_{i=1}^{n} \beta_{i} \phi_{i}^{-\zeta}\right) / \alpha\right]^{\frac{1}{\zeta}}
$$

Substituting (12) into (11), we can get the expression (13) on steady-state consumption growth rate which excludes $\mathrm{k}$ as expressed by $\eta$ :

$$
\eta=\frac{\alpha(1-\tau)\left\{\alpha \tau^{\zeta} / \tau^{\zeta}-\sum_{i=1}^{n} \beta_{i} \phi_{i}^{-\zeta}\right\}^{-\frac{(1+\zeta)}{\zeta}}-\rho}{\sigma}
$$

Based on equation (13), we make derivation of $\eta$ and can get the relationship between the share of item $i$ of public expenditure in total expenditure and the steady-state growth rate, as is shown in equation (14):

$$
\frac{\partial \eta}{\partial \phi_{i}}=\frac{\alpha(1-\tau)(1+\zeta)\left(\alpha \tau^{\zeta}\right)^{-\frac{(1+\zeta)}{\zeta}}\left[\beta_{i} \phi_{i}^{-(1+\zeta)}-\beta_{j} \phi_{j}^{-(1+\zeta)}\right]}{\sigma\left[\tau^{\zeta}-\sum_{i=1}^{n} \beta_{i} \phi_{i}^{-\zeta}\right]^{-\frac{1}{\zeta}}}
$$

According to equation (14), we define productive and non-productive expenditures. Productive expenditure refers to an item of expenditure that the increase of its proportion can promote long-term growth rate, i.e. $\partial \eta / \partial \emptyset j>0$. On the contrary, non-productive expenditure refers to an item of expenditure that the increase of its proportion cannot promote long-term growth rate. When do we have $\partial \eta / \partial \emptyset \mathrm{j}>0$ ? Based on the value range of each parameter, it can be known that for equation (14), if and only if $\beta_{\mathrm{i}} \phi_{\mathrm{i}}^{-(1+\zeta)}-\beta \mathrm{j} \phi \mathrm{j}^{-(1+\zeta)}>0$ there is $\partial \eta / \partial \emptyset \mathrm{j}>0$ and accordingly the condition of productive expenditure can be obtained, as is shown in equation (15):

$$
\left(\frac{\beta_{i}}{\beta_{j}}\right)^{\theta} \succ \frac{\phi_{i}}{\phi_{j}}
$$

Where $\theta=1 / 1+\zeta$ is the element's elasticity of substitution of production function.

When $\left(\beta_{\mathrm{i}} / \beta \mathrm{j}\right)^{\theta}$ is greater than $\emptyset_{\mathrm{i}} / \varnothing_{\mathrm{j}}$, improving the proportion of item $\mathrm{i}$ in public expenditure by reducing the proportion of item $\mathrm{j}$ in public expenditure can promote the increase of long-term economic growth rate until $\left(\beta_{\mathrm{i}} / \beta_{\mathrm{j}}\right)^{\theta}$ is equal to $\emptyset_{\mathrm{i}} / \varnothing_{\mathrm{j}}$, It can be seen that $\left(\beta_{\mathrm{i}} / \beta_{\mathrm{j}}\right)^{\theta}$ being equal to $\emptyset_{\mathrm{i}} / \emptyset_{\mathrm{j}}$ is a critical value, the economic significance of $\left(\beta_{\mathrm{i}} / \beta_{\mathrm{j}}\right)^{\theta}$ being equal to $\emptyset_{\mathrm{i}} / \varnothing_{\mathrm{j}}$ lies in that item $\mathrm{i}$ and item $\mathrm{j}$ expenditure have reached the optimal structure by this time. Therefore we call equation (16) as the optimal structure formula for public expenditure.

$$
\left(\frac{\beta_{i}}{\beta_{j}}\right)^{\theta}=\frac{\phi_{i}}{\phi_{j}}
$$


In order to have a clearer view of the economic significance behind the formula, we assume $\zeta=0$. We know when the estimated value of $\zeta$ is 0 and element's elasticity of substitution $\theta$ is 1 , the production function of CES degenerates into Cobb-Douglas production function. The conditions to be met with by productive expenditure become equation (17).

$$
\frac{\beta_{i}}{\beta_{j}} \succ \frac{\phi_{i}}{\phi_{j}}
$$

The formula of optimal structure of public expenditure is equation (18).

$$
\frac{\beta_{i}}{\beta_{j}}=\frac{\phi_{i}}{\phi_{j}}
$$

By model derivation analysis, we can make some tentative conclusions about the relationship between public expenditure structure and economic growth in the situation where total public expenditure remains constant, which are specifically summarized as follows: The impact of public expenditure structure on economic growth rate depends not only on the output elasticity of various expenditures $\beta_{\mathrm{i}}$, but also on the initial shares of various expenditures $\emptyset_{\mathrm{i}}$. The output elasticity of one item of public expenditure being greater than that of another item cannot guarantee that economic growth will be brought by reducing other items of public expenditure to increase this item. When an item of public expenditure has great output elasticity but its initial share in total public expenditure is oversized, it will not promote economic growth by reducing other expenditure to increase the proportion of this item, i.e., because of the oversized initial share, an item of expenditure which appears to be productive will become non-productive; there exists optimal ratio of public expenditure in economic system, the formula of which is $\left(\beta_{\mathrm{i}} / \beta_{\mathrm{j}}\right)^{\theta}=\emptyset_{\mathrm{i}} / \varnothing_{\mathrm{j}}$, and $\beta_{\mathrm{i}} / \beta_{\mathrm{j}}=\emptyset_{\mathrm{i}} / \emptyset_{\mathrm{j}}$ when $\theta$ is 1 . On this condition, we cannot promote economic growth by reducing the proportion of one item of expenditure to increase that of another item.

At this point, we have extended Shantayanan Devarajan,Vinaya Swaroop and Heng-fu Zuo's model of the impact of public expenditure structure on economic growth from one including two components of expenditure to a model including $\mathrm{n}$ components. We have demonstrated the prerequisites of promoting economic growth by adjusting public expenditure structure, on the condition of not altering the total scale of public expenditure. Compared with the model of two components, the model extended to $\mathrm{n}$ components has arrived at consistent conclusions, i.e. the impact of public expenditure structure on economic growth not only depends on the marginal output elasticity of each item of expenditure, but also on their initial shares.

\section{Empirical Test}

Our empirical research is the analysis of the relationship between public expenditure structure and economic growth based on data from China.

\subsection{Data and Variables}

We divide public expenditure into four dimensions according to functional structure, i.e. economic construction expenditure, social expenditure on culture and education, defense expenditure and administrative expenditure. Econ/Te, Social/Te, Def/Te and Admi/Te mean respectively the proportions of economic construction expenditure, social expenditure on culture and education, defense expenditure and administrative expenditure in total public expenditure. The scale of public expenditure is represented by the proportion of total public expenditure in GDP (Te/GDP). The role of labor in economic growth is represented by the growth rate of working population (GRPopu). Economic growth is represented by the growth rate of per capita GDP (GRPCGDP).

Due to the altered statistical coverage of public expenditure in 2007 by the Chinese Government, data after 2007 cannot be compared with those before 2007. Consequently considering the data availability and consistency of statistical coverage, we choose the time series data from 1978 to 2006, all of which are obtained from China Statistical Yearbook 1978-2006.

Firstly, we conduct an ADF test on the time series data. The detailed test results are reported in Table 1. The ADF values of variables in the table are all below the $95 \%$ critical value, showing that the null hypothesis is rejected at $5 \%$ significance level. All the time series are variables of integrated of order zero, i.e. stationary time series. Therefore we can directly adopt the original variables to establish VAR model. 
Table 1. ADF test results

\begin{tabular}{ccccc}
\hline Variables & $(\mathrm{C}, \mathrm{d}, \mathrm{T})$ & ADF & Critical Value (99\%) & Critical Value (95\%) \\
\hline Econ/Te & $(0,0,0)$ & $-4.345607^{* * *}$ & -2.650145 & -1.953381 \\
Social/Te & $(\mathrm{C}, 0,0)$ & $-4.365975^{* *}$ & -3.689194 & -2.971853 \\
Def/Te & $(\mathrm{C}, 0, \mathrm{~T})$ & $-6.245495^{* * *}$ & -4.416345 & -3.622033 \\
$\mathrm{Admi} / \mathrm{Te}$ & $(\mathrm{C}, 0, \mathrm{~T})$ & $-3.597291^{* *}$ & -4.339330 & -3.587527 \\
$\mathrm{Te} / \mathrm{GDP}$ & $(\mathrm{C}, 0,0)$ & $-3.612278^{* *}$ & -3.699871 & -2.976263 \\
GRPopu & $(\mathrm{C}, 0, \mathrm{~T})$ & $-5.511608^{* * *}$ & -4.323979 & -3.580623 \\
GRPCGDP & $(\mathrm{C}, 0,0)$ & $-3.714983^{* *}$ & -3.724070 & -2.986225 \\
\hline
\end{tabular}

Note. In the following text, all the *** represents $1 \%$ significance level; ** represents $5 \%$ significance level; *represents $10 \%$ significance level.

\subsection{Regression Analysis: Analysis Based on VAR Model}

We choose the lag order of the model according to LR test methods and establish model VAR (1). The model has passed the joint significance test of coefficients of all orders, and the residual is white noise. Moreover, this VAR system is stable. As in Figure1 Roots of the Companion Matrix, all the eigenvalues of the model is within the unit root.

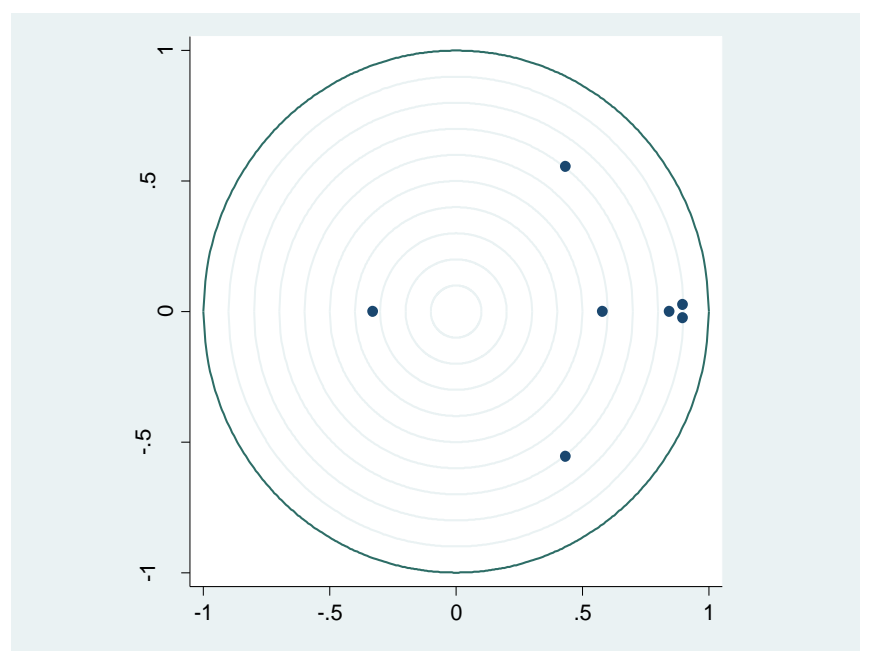

Figure1. Roots of the companion matrix

Then estimate the causality between public expenditure structure and changes of economic growth rate based on the Granger Causality Test. As in Table 2, in the relationship between public expenditure structure and economic growth, there exists a unidirectional causality between the growth rate of per capita GDP and the proportions of economic construction expenditure, social expenditure on culture and education, and administrative expenditure. In other words, the changes of the growth rate of per capita GDP is caused by the changes of the proportions of economic construction expenditure, social expenditure on culture and education, administrative expenditure, and not the other way round.

Table 2. The Granger Causality test results

\begin{tabular}{lccc}
\hline Null Hypothesis & F Statistic & DOF & P Value \\
\hline Econ/Te does not Granger Cause GRPCGDP & 3.4865 & 1 & $0.0766^{*}$ \\
Social/Te does not Granger Cause GRPCGDP & 4.6478 & 1 & $0.0434^{* *}$ \\
Admi/Te does not Granger Cause GRPCGDP & 4.6308 & 1 & $0.0438^{* *}$ \\
Te/GDP does not Granger Cause GRPCGDP & 4.1172 & 1 & $0.056^{*}$ \\
GRPopu does not Granger Cause GRPCGDP & 3.2003 & 1 & $0.0888^{*}$ \\
All variables joint together does not simultaneously Granger Cause GRPCGDP & 2.7105 & 6 & $0.0431^{* *}$ \\
\hline
\end{tabular}


In what follows, we will build impulse response functions to analyze the dynamic relationship between public expenditure structure and economic growth system as well as the dynamic impact on economic growth by economic construction expenditure, social expenditure on culture and education, defense expenditure and administrative expenditure.

According to the impulse response function of per capita GDP to Econ/Te, we have obtained the dynamic time trajectory of the changes in per capita GDP triggered by one standard deviation shock in Econ/Te. As in Figure 2, the impulse response curve of Econ/Te to GRPCGDP has a significant valley. When the shock in Econ/Te occurs, GRPCGDP shows an obvious negative response and drops to the bottom in the second stage. Then the negative shock begins to weaken. Viewing from cumulative response as in table3, when the shock in Econ/Te occurs, GRPCGDP has a cumulative and continuous negative response and drops to the bottom in the fourth stage.

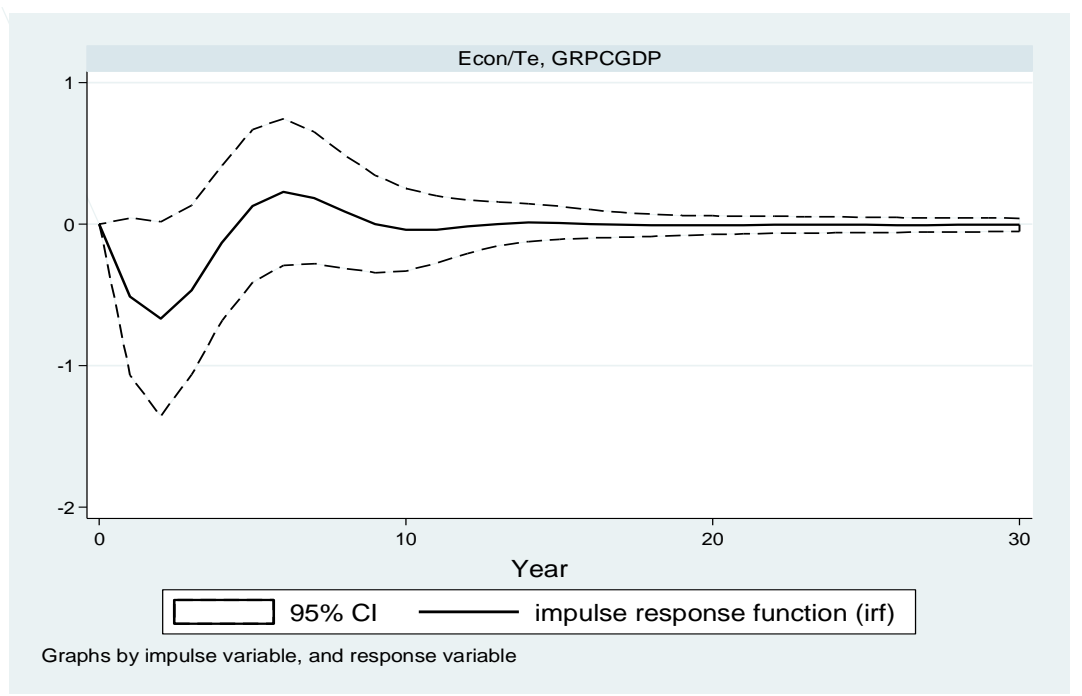

Figure 2. The graphs of impulse response function of GRPCGDP to Econ/Te

As in Figure 3, the impulse response curve of Social/Te to GRPCGDP has a significant crest. When the shock in Social/Te occurs, GRPCGDP shows an obvious positive response and reaches the top in the first stage. Then the positive shock begins to weaken. Viewing from cumulative response as in table3, when the shock in Social/Te occurs, GRPCGDP has a cumulative and continuous positive response and reaches the top in the seventh stage.

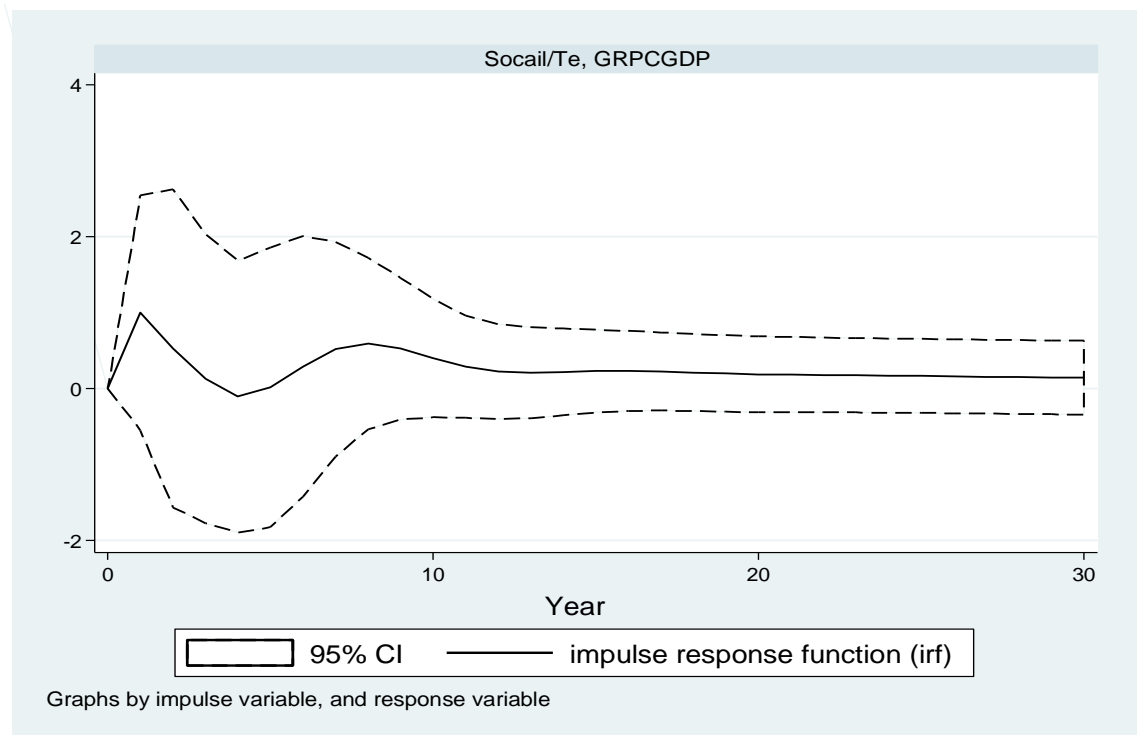

Figure 3. The graphs of impulse response function of GRPCGDP to Social/Te 
As in Figure 4, the impulse response curve of GRPCGDP to Admi/Te has a significant valley. When the shock in Admi/Te occurs, GRPCGDP shows an obvious negative response and reaches the top in the second stage. Then the negative shock begins to weaken. Viewing from cumulative response as in table3, when the shock in Admi/Te occurs, GRPCGDP has a cumulative and continuous negative response and reaches the top in the third stage.

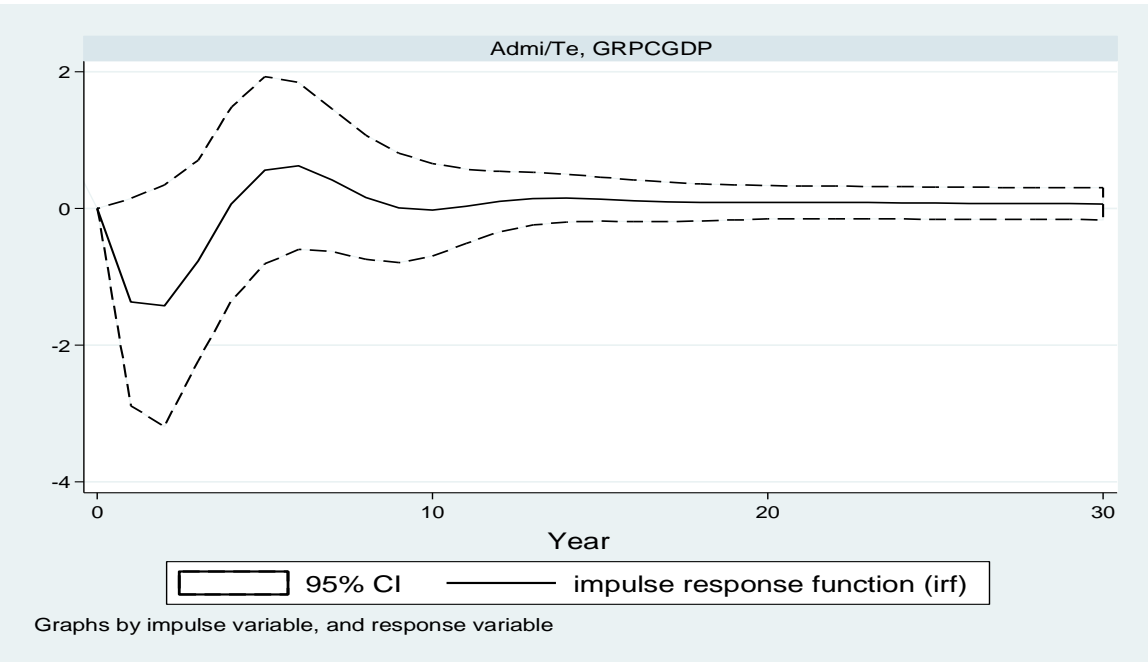

Figure 4. The graphs of impulse response function of GRPCGDP to Admi/Te

As in Figure 5, the impulse response curve of Def/Te to GRPCGDP has a significant crest. When the shock in Def/Te occurs, GRPCGDP shows an obvious positive response and reaches the top in the first stage. Then the positive shock begins to weaken. Viewing from cumulative response, when the shock in Def/Te occurs, GRPCGDP has a cumulative and continuous positive response and reaches the top in the eighth stage.

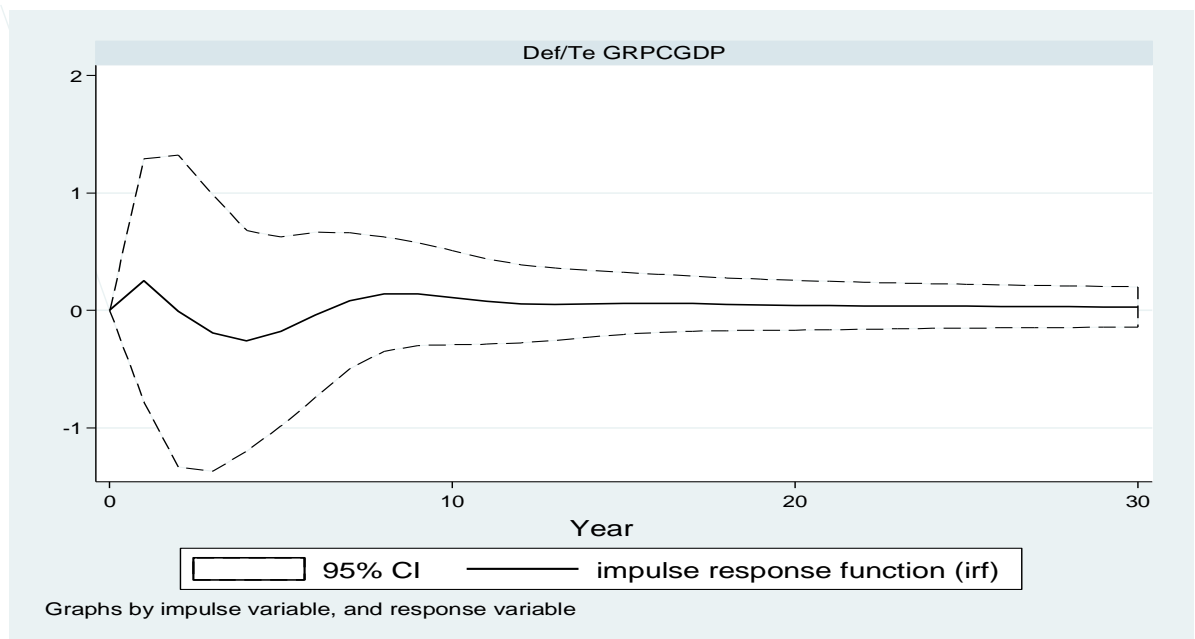

Figure 5. The graphs of impulse response function of GRPCGDP to Def/Te

Table 3 is a summary of the impulse response values of public expenditure structure to GRPCGDP. Based on VAR model, we draw the following main conclusions: Econ/Te has a cumulative negative impact on economic growth; Social/Te has a cumulative positive impact on economic growth; Admi/Te has a cumulative negative impact on economic growth; Def/Te has a cumulative positive impact on economic growth; there exists a significant unidirectional causality between economic growth and Econ/Te, Social/Te and Admi/Te, their changes are the reasons for changes in economic growth; there is no significant causality between economic growth and Def/Te, to some extent Def/Te is an exogenous variable of the economic growth. 
Table 3. Impulse response values of public expenditure structure to GRPCGDP

\begin{tabular}{|c|c|c|c|c|c|c|c|c|}
\hline & \multicolumn{2}{|c|}{$\begin{array}{c}\text { Economic Construction } \\
\text { Expenditure }\end{array}$} & \multicolumn{2}{|c|}{$\begin{array}{l}\text { Social Expenditure on } \\
\text { Culture and Education }\end{array}$} & \multicolumn{2}{|c|}{ Defense Expenditure } & \multicolumn{2}{|c|}{ Administrative Expenditure } \\
\hline & Current Stage & Cumulative & Current Stage & Cumulative & Current Stage & Cumulative & Current Stage & Cumulative \\
\hline 1 & -0.5050 & -0.5050 & 1.2578 & 1.2578 & 0.3189 & 0.3189 & -1.4951 & -1.4951 \\
\hline 2 & -0.6104 & -1.1154 & 0.8121 & 2.0698 & 0.1291 & 0.4480 & -1.4383 & -2.9334 \\
\hline 3 & -0.3491 & -1.4644 & 0.0571 & 2.1269 & -0.0247 & 0.4233 & -0.4211 & -3.3545 \\
\hline 4 & -0.0373 & -1.5017 & -0.2665 & 1.8604 & -0.0625 & 0.3608 & 0.4692 & -2.8854 \\
\hline 5 & 0.1309 & -1.3708 & -0.0924 & 1.7680 & 0.0047 & 0.3655 & 0.7787 & -2.1067 \\
\hline 6 & 0.1346 & -1.2361 & 0.2605 & 2.0285 & 0.0800 & 0.4455 & 0.6129 & -1.4939 \\
\hline 7 & 0.0619 & -1.1742 & 0.5030 & 2.5315 & 0.1091 & 0.5546 & 0.3163 & -1.1776 \\
\hline 8 & 0.0005 & -1.1737 & 0.5470 & 3.0785 & 0.0898 & 0.6444 & 0.1346 & -1.0430 \\
\hline
\end{tabular}

Note. First column means time intervals, which is measured by year.

\subsection{Robust Test}

In order to retest the relationship between public expenditure structure and economic growth, we adopt the general econometric model and establish a regression equation as in equation (19).

$$
G R P C G D P_{(t+1, t+n)}=\alpha_{1}+a_{2} G R P o p u+\alpha_{3}(T e / G D P)+\sum_{k} \alpha_{k}(G / T E)_{t}+\mu_{t}
$$

The explained variable GRPCGDP $(t+1, t+n)$ in equation (19) is the moving average of GRPCGDP which moves forward to $\mathrm{n}$ stages. The reasons for choosing the moving average which moves forward to $\mathrm{n}$ stages are as follows: (a) the effect of public expenditure items on economic growth has a certain time lag and average by moving forward can mitigate the impact of short-term fluctuations; (b) to solve the endogeneity and reverse causality of public expenditure structure. For example, if there is a reverse relationship between social expenditure on culture and education and the current economic growth, it does not mean social expenditure on culture and education has a negative impact on economic growth. It is probably because of the relatively low economic growth rate, the government increases social expenditure on culture and education in the current stage with an aim to promote economic growth. Therefore we think that the public expenditure structure of $t$ stage has an impact on the moving average from stage $t+1$ to $t+n$. $(\mathrm{G} / \mathrm{TE})_{t}$ is the vector of public expenditure structure, which includes Econ/Te, Social/Te, Def/Te and Admi/Te. As a controlled variable, Te/GDP is the proportion of total public expenditure in GDP, GRPopu is the growth rate of working population and reflects the effect of changes in working population on GRPCGDP.

Table 4. OLS analysis of the relationship between public expenditure structure and economic growth

\begin{tabular}{ccc}
\hline & Eq. $(1.1)$ & Eq. $(1.2)$ \\
\hline Econ/Te & $-0.2207284^{*}$ & -0.1010963 \\
& $(-1.90)$ & $(-1.14)$ \\
Social/Te & $0.5880583^{* *}$ & $0.495894^{* *}$ \\
& $(2.12)$ & $(2.33)$ \\
Def/Te & $0.5153833^{* *}$ & $0.4762901^{* * *}$ \\
& $(2.49)$ & $(3.01)$ \\
Admi/Te & -0.3593504 & -0.0621185 \\
& $(-1.18)$ & $(-0.27)$ \\
Te/GDP & $0.2510352^{* * *}$ & $0.2355969 * * *$ \\
& $(2.28)$ & $(2.80)$ \\
GRPopu & $0.1952023 * *$ & $0.1496647 * *$ \\
& $(2.78)$ & $(2.79)$ \\
Constant & -0.072662 & -0.0684862 \\
R-Squared & $(-0.05)$ & $(-0.61)$ \\
Adj R-Squared & 0.6431 & 0.6772 \\
F value & 0.5457 & 0.5892 \\
\hline
\end{tabular}

Note. t-statistics in parentheses. 
As in Table 4, column (1.1) to column (1.2) reflect respectively the regression results of the moving average of public expenditure structure and GRPCGDP which move forward by 5 stages and 6 stages in China. To be specific: Econ/Te has a significant negative effect on economic growth. For each increase of Econ/Te by 1 percentage point, GRPCGDP drops by 0.22 percentage point. Social/Te has a significant positive effect on economic growth. For each increase of Social/Te by 1 percentage point, GRPCGDP increases by 0.59 percentage point. Admi/Te has a negative but not significant effect on long-term economic growth in China. Def/Te has a significant positive effect on economic growth in China.

No matter by VAR model or by classic single equation econometric model, we have all obtained the same relationship between economic growth and Econ/Te, Social/Te and Admi/Te, i.e., in the situation of a constant total scale of public expenditure, Econ/Te has a negative effect on economic growth; Social/Te has a positive effect on economic growth. This result is contrary to the results obtained by Barro, but consistent with the structure obtained by Shantayanan Devarajan, Vinaya Swaroop and Heng-fu Zou with analysis of data from 43 developing countries. By empirical test, we also draw the conclusion that Admi/Te has a negative effect on economic growth and Def/Te has a positive effect.

\section{Explanations}

Usually people tend to think that one of the reasons for the rapid economic growth in China over the past three decades is the large-scale economic construction expenditure. However the results of empirical test show that Econ/Te has a negative correlation with economic growth and Social/Te has a positive correlation with economic growth. If we look back to the theoretical model, we will find that it is not surprising to have obtained such results. Because Econ/Te is too high in the public expenditure structure in China, it becomes non-productive. On the contrary, Social/Te is too low in the public expenditure structure in China, it becomes productive. Taking China and OECD countries as examples, the average Econ/Te of China is $44.2 \%$ while the Econ/Te of OECD countries mostly keeps stable between $10 \%$ and $15 \%$. Contrary to Econ/Te, the Social/Te of OECD countries have all passed 50\% and settled at around 60\%. Taking the Unite States as an example, its Social/Te stays at around 55\% for a long time. The Social/Te of welfare states such as Denmark and the Netherlands reaches $70 \%$. And the Social/Te of China is only $23.5 \%$.

Besides, the following are also the reasons for the significant negative impact of Econ/Te on economic growth. Firstly, viewing from the practical situation in China, the low efficiency of economic construction expenditure is common. What always go together with the expansion of economic construction expenditure are the blind investment, repeated constructions and rent-seeking corruptions of some local governments. The low efficiency or even inefficiency of economic construction expenditure has restricted its positive effect. Secondly, economic construction expenditure as government investment has Crowding-out Effect on private investment. In the relatively short economic recession, government investment does not have Crowding -out Effect on private investment; instead it even has Crowding In Effect. But in the long run, the Crowding-out Effect of government investment on private investment becomes significant. The reform and opening-up of China from 1978 started with the change from government-led planned economy to government-led market economy. On the one hand, government-led market economy has played a positive role in gathering strengths; one the other hand, in the process of resource allocation, the right boundaries of the government are too broad. Governments at various levels have undertaken excessive and direct economic development functions, which has a negative impact on private production. Meanwhile, too much economic construction expenditure leads to the risk accumulation of local government debts, bringing unstable factors to the operation of macro economy. The positive correlation between social expenditure on culture and education and economic growth is also because that social expenditure on culture and education can have positive impact on the labor and technology. It can promote the accumulation of human capital and progress of science and technology, and hence have a positive impact on long-term economic growth. The reasons for the negative impact of Admi/Te lie in the following: Firstly, there are numerous administrative institutions with large number of staff in China; secondly, the usage efficiency of administrative resource is not high and there is waste; thirdly, the operation efficiency of administrative institutions is not high. Administrative expenditure is, ultimately, non-productive expenditure. As an item of complete consumption expenditure, it does not produce material wealth directly, and crowds out the input of public expenditure in public service area and productive areas.

\section{Conclusions and Suggestions}

The theoretical model established in this paper shows that the impact of public expenditure structure on economic growth rate depends not only on the output elasticity of various items of expenditure, but also on the initial shares of them. The output elasticity of one item of public expenditure being greater than that of the other 
public expenditure cannot guarantee that increasing this expenditure by reducing other items of expenditure will bring about economic growth. When one item of expenditure has big output elasticity but its initial share in total public expenditure is oversized, economic growth will not be promoted by reducing other expenditure to increase the proportion of this expenditure.

The research results have important policy implications for the Chinese Government in its future adjustment of public expenditure structure and determination of the focus of public expenditure. Firstly, Econ/Te should be reduced and the problem of financial offside be solved. The input direction of funds should be optimized and economic construction expenditure be more directed to infrastructure construction, creating good environment for economic development. Economic construction expenditure should withdraw from general competitive areas and production and circulation processes, and play more of the function of public service. The evaluation and motivation targets of government officials should be changed and the investment impulsion of local governments be restrained. Secondly, Social/Te should be increased and the problem of financial vacancy be solved. Public educational expenditure should be increased and the public nature of educational expenditure be highlighted. The input of public expenditure in scientific researches should be enhanced, thus promoting the economic development by scientific and technological innovations. The input of public expenditure in healthcare should be increased so as to ease the tension in medical resources. The level of social welfare expenditure should be improved and social welfare system be strengthened. Thirdly, Admi/Te should be controlled and the problem of redundancy be solved. The boundaries of government functions should be made clear and "offside" be reduced. Institutional reform should be promoted and the redundancy be cut. The operation efficiency of administrative expenses should be improved and the administrative cost be reduced, so as to provide a good environment for economic and social development. Fourthly, the supervision and administration of public expenditure should be enhanced and the efficiency of funds usage be improved. Attention should be paid to the administration of public expenditure. The administration system of public expenditure which is "standard, highly efficient and sound" should be established. The system of open finance should be promoted and perfected, and the transparency of public expenditure should be continuously improved. The mechanisms of public expenditure in terms of supervision, evaluation and public decision making should be improved and the supervision of financial funds be enhanced. Fifthly, the adjustment direction of public expenditure structure being reformed from economic-construction finance to public finance should be adhered to. Opinions should be changed and the approach of economic-construction finance should be transformed to public finance and finance of people's livelihood. Taking the satisfaction of social public needs as the goal, the boundaries of government functions and duties should be reasonably defined. The decisive role of market in resource allocation should be played and market rules, market prices and market competition be taken as the main adjustment methods of micro-level resource allocation.

\section{References}

Arrow, K. J., \& Kurz, M. (1970). Optimal Growth with Irreversible Investment in a Ramsey Model. Econometric, 38(2), 331-344. http://doi.org/10.2307/1913014

Barro, R. J. (1981). Output Effects of Government Purchases. Journal of Political Economy, 89(6), 1086-1121. http://dx.doi.org/10.1086/261024

Barro, R. J. (1990). Government Spending in a Simple Model of Endogeneous Growth. Journal of Political Economy, 98(5), S103-S125. http://dx.doi.org/10.1086/261726

Blankenau, W. F., Simpson, N. B., \& Tomljanovich, M. (2007). Public Education Expenditures, Taxation, and Growth: Linking Data to Theory. The American Economic Review, 97(2), 393-397. http://dx.doi.org/10.1257/aer.97.2.393

Chen, B. L. (2006). Economic Growth with an Optimal Public Spending Composition. Oxford Economic Papers, 58(1), 123-136. http://dx.doi.org/10.1093/oep/gpi045

Fei-yue, G., \& Feng-zhi, X. (2012). The Empirical Research on China's Government Expenditure Structure and Economic Growth. Current Economic Information, 9, 88-90.

MacDonald, L. (2008). The Impact of Government Structure on Local Public Expenditures. Public Choice, 136(3/4), 457-473. http://dx.doi.org/10.1007/s11127-008-9308-8

Ramirez, M. D. (1997). Review of Economic Policy and Stabilization in Latin America. Southern Economic Journal, 64(1), 360-362. http://doi.org/10.2307/1061080

Sanz, I. (2011). What Do OECD Countries Cut First When Faced with Fiscal Adjustments. Southern Economic Journal, 77(3), 753-775. http://dx.doi.org/10.4284/sej.2011.77.3.753 
Sanz, I., \& Velázquez, F. J. (2004). The Evolution and Convergence of the Government Expenditure Composition in the OECD Countries. Public Choice, 119(1/2), 61-72. http://dx.doi.org/10.1023/B:PUCH.0000024162.20553.8e

Shang-zhi, Z. (2012). The Empirical Analysis on the Impact of Fiscal Expenditure Structure Distort on Economic Growth. Review of Economics and Management, 7, 87-91.

Shantayanan, D., Vinaya, S., \& Heng-fu, Z. (1996). The Composition of Public Expenditure and Economic Growth. Journal of Monetary Economics, 37, 313-344. http://dx.doi.org/10.1016/S0304-3932(96)90039-2

Woodford, M. (2011). Simple Analytics of the Government Expenditure Multiplier. American Economic Journal: Macroeconomics, 3(1), 1-35. http://dx.doi.org/10.1257/mac.3.1.1

\section{Copyrights}

Copyright for this article is retained by the author(s), with first publication rights granted to the journal.

This is an open-access article distributed under the terms and conditions of the Creative Commons Attribution license (http://creativecommons.org/licenses/by/3.0/). 